\title{
Anisotropic magnetization-induced second harmonic generation in Fe/Au superlattices
}

\author{
K. Sato, A. Kodama, and M. Miyamoto \\ Tokyo University of Agriculture and Technology, Koganei, Tokyo 184-8588, Japan \\ A. V. Petukhov* \\ Physical and Colloid Chemistry, University of Utrecht, Padualaan 8, 3584 CH Utrecht, The Netherlands \\ K. Takanashi, S. Mitani, and H. Fujimori \\ Institute for Materials Research, Tohoku University, Sendai 980-8577, Japan \\ A. Kirilyuk ${ }^{\dagger}$ and Th. Rasing \\ Research Institute for Materials, University of Nijmegen, Toernooiveld 1, 6525 ED Nijmegen, The Netherlands
}

(Received 2 April 2001; published 22 October 2001)

\begin{abstract}
The anisotropic nonlinear magneto-optical response from $\mathrm{Fe} / \mathrm{Au}(001)$ superlattices is studied. Perfect singlecrystalline order through the whole thickness of the multilayer is observed. The magneto-optical signals are measured in both the longitudinal and transversal configurations. A strong fourfold anisotropy of nonlinear Kerr rotation angle is demonstrated, in addition to the anisotropic second harmonic intensity changes. The results are described in terms of a simple phenomenological model, that involve both dipole and quadrupole nonlinear-optical interactions. Furthermore, general, model-independent symmetry properties of the nonlinear magneto-optical response are established.
\end{abstract}

DOI: 10.1103/PhysRevB.64.184427

PACS number(s): 75.70.-i, 78.20.Ls, 42.65.Ky

\section{INTRODUCTION}

Nonlinear magneto-optics is attracting attention as a novel tool for the investigation of surfaces and interfaces of magnetic materials. Among the different nonlinear magnetooptical effects, the most intensively studied is the magnetization-induced second harmonic generation (MSHG). ${ }^{1-5}$ This effect is known to be very sensitive to surfaces and interfaces, since in centrosymmetric materials the electric dipole-induced SHG becomes allowed only at surfaces and interfaces where the inversion symmetry is broken. In addition, the magnetization also lowers the symmetry at the surfaces and interfaces. This is the reason why MSHG has been applied to studies of magnetic thin films and multilayers. ${ }^{6-10}$

In most cases, isotropic MSHG from magnetic metal interfaces has been studied. The only investigated cases of anisotropic MSHG from single crystalline samples were noncentrosymmetric films of magnetic garnets $^{11}$ and antiferromagnetic crystals. ${ }^{4,5}$ These early anisotropic studies were fully described by the simplest dipole approximation. Except in the special case of domain wall effects, ${ }^{12}$ higherorder contributions were not taken into account.

In this study we have applied the MSHG technique to $\mathrm{Fe} / \mathrm{Au}$ superlattices with atomically controlled epitaxial layers. The superlattice with a modulation of mono-atomic layers of $\mathrm{Fe}$ and $\mathrm{Au}$ has been known to show an artificial order with an $\mathrm{L} 1_{0}$ structure that does not exist in nature. ${ }^{13}$ Such an artificial structure remains at interfaces between $\mathrm{Fe}$ and $\mathrm{Au}$ layers when the modulation period becomes longer than monoatomic. ${ }^{14}$ The linear magneto-optical spectra of the superlattices modulated by integer and non-integer numbers of atomic layers have been studied intensively, suggesting the formation of a peculiar band structure in such an artificial real-space structure. ${ }^{15,16}$

Strong second harmonic (SH) signals were observed from the $\mathrm{Fe} / \mathrm{Au}$ superlattices that showed clear fourfold anisotropy in the azimuthal dependence of $\mathrm{SH}$ intensity and the magnetization-induced effect, which demonstrates a high long-range in-plane order in the superstructures. The azimuthal dependence of the nonlinear magneto-optical Kerr rotation has also been studied. The results are analyzed on the basis of phenomenological considerations including dipole as well as quadrupole contributions to the MSHG response. It is shown that the minimum set of contributions to describe the data involve interface nonmagnetic, interface magnetic and the quadrupole nonmagnetic interactions. We also discuss a general, model-independent symmetry of the MSHG response that reveals the sample symmetry.

\section{GENERAL THEORY}

An incident light wave induces a polarization in a medium that serves as a source for the transmitted and reflected light. The polarization $\mathbf{P}$ can be written as an expansion in powers of the optical electric field $\mathbf{E}(\omega)$ :

$$
\begin{aligned}
\mathbf{P}(\omega, 2 \omega, \ldots)= & \chi^{1, d} \mathbf{E}(\omega)+\chi^{1, q} \boldsymbol{\nabla} \mathbf{E}(\omega)+\chi^{2, d} \mathbf{E}(\omega) \mathbf{E}(\omega) \\
& +\chi^{2, q} \mathbf{E}(\omega) \boldsymbol{\nabla} \mathbf{E}(\omega) \ldots
\end{aligned}
$$

The tensor $\hat{\chi}^{1}$ is the linear optical susceptibility allowed in all media. SHG is described by the third and the fourth term where the electric-dipole tensor $\chi^{2, d}$ is allowed only in noncentrosymmetric media and on surfaces and interfaces, while the quadrupole tensor $\chi^{2, q}$ is allowed everywhere. For crystals with a spontaneous or magnetic-field induced magneti- 
zation $\mathbf{M}$, expansion of the nonlinear optical polarization of a medium $\mathbf{P}^{n l}(2 \omega)$ can be further written (keeping only linear in magnetization terms) as

$$
\begin{aligned}
\mathbf{P}^{n l}(2 \omega)= & \chi^{c r} \mathbf{E}(\omega) \mathbf{E}(\omega)+\chi^{m} \mathbf{E}(\omega) \mathbf{E}(\omega) \mathbf{M} \\
& +\chi^{q, c r} \mathbf{E}(\omega) \nabla \mathbf{E}(\omega)+\chi^{q, m} \mathbf{E}(\omega) \nabla \mathbf{E}(\omega) \mathbf{M},
\end{aligned}
$$

where the first and third term describe the purely crystallographic contribution while the second and fourth only exists in the presence of a magnetization $\mathbf{M}$. The first two contributions to the nonlinear polarization $\mathbf{P}^{n l}(2 \omega)$ are of electricdipole character and therefore can appear only at the interfaces of centrosymmetric media. Their properties are however different. The crystallographic contribution is described by a polar tensor of rank 3 , whereas the magnetization-induced contribution is described by an axial tensor of rank 4. When allowed, the interference between these terms can give rise to new nonlinear magneto-optical effects which have no counterparts in linear optics. ${ }^{11,17}$

Although smaller, the last two terms in Eq. (2) originate from the bulk and therefore may be comparable in magnitude to the strong dipole contribution coming from the very thin interface layer. The experimental results (see below) confirm this assumption and substantiate the necessity to take this contribution into account in high-quality single-crystalline multilayers.

Further theoretical considerations are presented in the Appendix; the results are used to describe the experimental results of Secs. IV and V.

\section{EXPERIMENT}

The samples used in the present study are the same ones as used in the previous linear magneto-optical studies. ${ }^{16}$ They were prepared on $\mathrm{MgO}$ (100) substrates by an ultrahigh vacuum (UHV) deposition technique. The base pressure of the deposition system was $3 \times 10^{-10}$ Torr. An Fe seed layer of $1 \mathrm{~nm}$ followed by a Au buffer layer of $50 \mathrm{~nm}$ was deposited at $200^{\circ} \mathrm{C}$ and subsequently annealed for $30 \mathrm{~min}$ to $1 \mathrm{~h}$ at $500^{\circ} \mathrm{C}$. The orientation of the Au buffer layer was $(001)$. The Fe seed was necessary to control the orientation of the $\mathrm{Au}$ layer. Multilayers with $N$ periods, each period consisting of $x$ monolayers (ML) of Fe and $x \mathrm{ML} \mathrm{Au}$, were deposited in the UHV system at $70^{\circ} \mathrm{C}$ on the Au buffer. The parameter $x$ took integer values $(x=1,2,3,4,5,6,8,10$ and 15$)$ or noninteger values between 1 and 4 . The number of periods $N$ was chosen in order to obtain the total multilayer thickness $\approx 200 \mathrm{ML}$ the same for all samples. The deposition rates were approximately $0.01 \mathrm{~nm} / \mathrm{s}$. The layer thickness was controlled using a quartz thickness monitor. Superlattices employed for the nonlinear magneto-optical measurements were those with $x=1,10$ and 15 ML. As a typical example of a noninteger superlattice, a sample with $x=3.5$ was also studied. Details of preparation techniques were described elsewhere. ${ }^{18}$ Formation of superlattice structure was confirmed by x-ray diffraction as described in detail in Ref. 16 .

MSHG measurements were performed at both the Re-

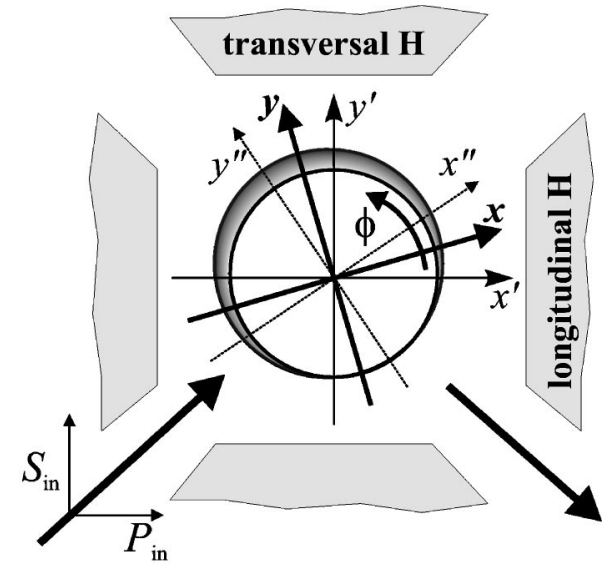

FIG. 1. Schematic experimental geometry: sample rotates azimuthally in the applied magnetic field, either in transversal or longitudinal magneto-optical configuration. $x y$ : sample frame (two equivalent symmetry planes); $x^{\prime} y^{\prime}$ : laboratory frame.

search Institute for Materials (RIM), University of Nijmegen, and Tokyo University of Agriculture and Technology (TUAT). We used a mode-locked Ti-sapphire laser (Coherent, MIRA, $\lambda=720-850 \mathrm{~nm})$ as a light source. The Tisapphire laser was excited by either a 514.5-nm line of a 8-W $\mathrm{Ar}^{+}$ion laser (RIM) or a 532-nm radiation of a 5-W diodepumped $\mathrm{YVO}_{4}-\mathrm{SHG}$ laser (Coherent, VERDI) (TUAT). The pulse width of the Ti-sapphire laser was 100-150 fs and the repetition rate was $80 \mathrm{MHz}$. The averaged power output of the laser was approximately $600 \mathrm{~mW}$. To avoid sample damage by the laser irradiation, the averaged power of the light beam was reduced to $1 / 10-1 / 20$ of the original intensity using a light chopper with a small duty cycle. The spot size of the laser beam focused on the sample was $40-80 \mu \mathrm{m}$ in diameter and the peak power density was estimated as $0.5-1 \mathrm{GW} / \mathrm{cm}^{2}$.

The incident angle of the laser beam was fixed at $45^{\circ}$ to the sample normal. Magnetic fields up to about 0.2-0.3 T were applied in both the longitudinal and transversal magneto-optical geometry (see Fig. 1). Magnetic hysteresis loops were measured separately using a vibrating sample

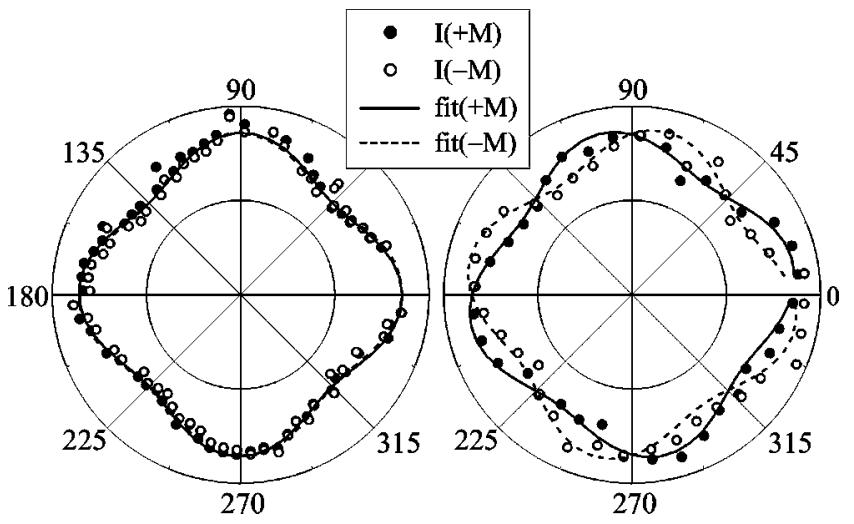

FIG. 2. Rotational anisotropy curves for samples with $x=1$ monolayers (left) and $x=15$ monolayers (right) in $P_{\text {in }} P_{\text {out }}$ polarization combination. 


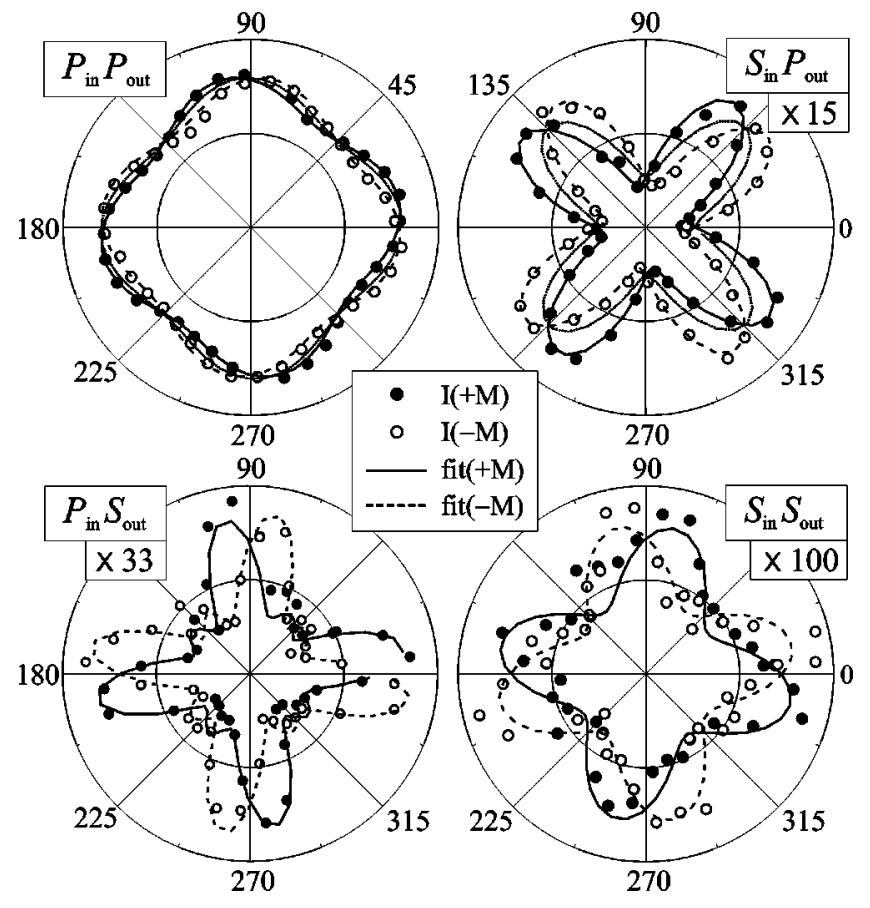

FIG. 3. Rotational anisotropy curves (experimental points plus theoretical fits) for the sample with single layer thickness $x=15$ monolayers in longitudinal geometry. Multiplication factors in three plots show the scaling of the corresponding data in order to reach the same intensity level as with the $P_{\text {in }} P_{\text {out }}$ polarization combination (this figure and the following two).

magnetometer (VSM). Samples with $x=3.5,10,15$ exhibited an in-plane magnetization, leading to an easy saturation with the applied magnetic field. On the other hand, the sample with $x=1$ ML showed a magnetization perpendicular to the plane and the field was found to be insufficient to saturate the magnetization in the sample plane.

The sample was mounted on a computer-controlled rotating stage to obtain the azimuthal angle dependence of the MSHG signal. The latter was measured for all the four combinations of input-output polarization; i.e., $P_{\text {in }} P_{\text {out }}, S_{\text {in }} P_{\text {out }}$, $P_{\text {in }} S_{\text {out }}, S_{\text {in }} S_{\text {out }}$, where the notations $P$ and $S$ denote the polarizations parallel and perpendicular to the incident plane of reflection, respectively (Fig. 1). For measurements of the nonlinear Kerr rotation a computer-controlled rotating analyzer was employed.

The SHG light was effectively filtered using two blue filters (Schott BG39) and detected by a photomultiplier (EMI 9863QA in RIM or Hamamatsu R464 in TUAT), the output of which was guided to a preamplifier (Stanford Research SR445 in RIM or Hamamatsu C5594 in TUAT) and a photon counting apparatus (Stanford Research SR400). Typical dataaccumulation time was $10 \mathrm{~s}$ per data point.

\section{ROTATIONAL MSHG INTENSITY PATTERNS IN Fe/Au SUPERLATTICES}

Figure 2 shows the results of the rotational anisotropy measurements for the $P_{\text {in }} P_{\text {out }}$ polarization combination for

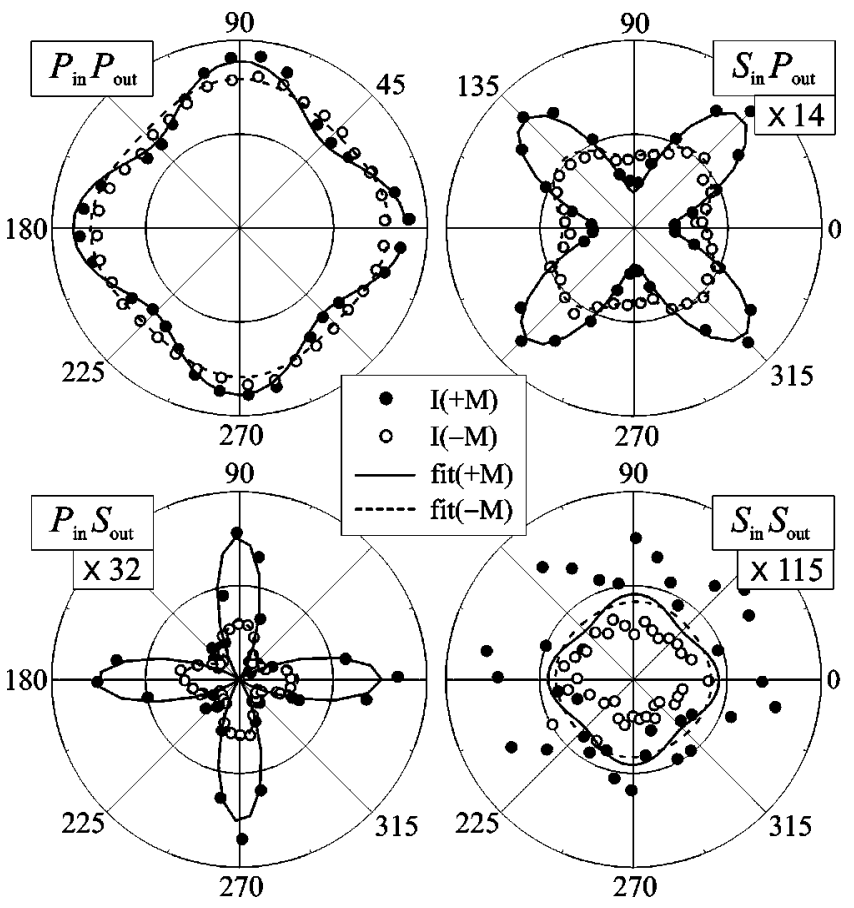

FIG. 4. Rotational anisotropy curves for the sample with single layer thickness $x=15$ monolayers in transversal geometry.

different repeat thicknesses ( $x=1$ and 15 ML). The both curves show a clear fourfold anisotropy. Only in the case with $x=15 \mathrm{ML}$, however, a clear magnetic contrast can be seen. The low magnetic contrast in the case with $x=1$ ML is most probably due to the insufficient saturation of the in-plane magnetization. Therefore further measurements were done with thicker samples only.

In Fig. 3, the results of all four polarization combinations for the sample with $x=15$ ML are plotted, for the longitudinal geometry. Note the different vertical scales for the various data, indicating a substantial difference for the MSHG response for different polarization combinations. It is also obvious that all data involving $S_{\text {in,out }}$ polarization yield a much stronger anisotropy, which is a direct consequence of the in-plane $x y$ tensor components that contribute to these signals (see below). Even the weakest $S_{\mathrm{in}} S_{\text {out }}$ curve shows a clear fourfold symmetry pattern.

For comparison, Fig. 4 shows the rotational anisotropy patterns for the same sample with $x=15 \mathrm{ML}$ in the transversal geometry. Qualitatively the difference in the magnetooptical effect is straightforward: while the pattern rotates in the longitudinal geometry (that actually corresponds to a polarization rotation similar to the linear case, see below), it shows just the intensity changes in the transversal one.

Usually, an analysis of MSHG results ${ }^{1,6,19}$ is performed assuming that the top surface and buried interfaces are the only sources of the nonlinear magneto-optical response. Their nonlinearity is described in terms of the effective surface/interface dipole-like nonlinear susceptibility $\chi^{(2)}(\mathbf{M})$, which is a third rank tensor. As discussed in more detail in Appendix A, this contribution yields the following azimuthal patterns: 
TABLE I. Azimuthal amplitudes $A^{\alpha, \beta}, B^{\alpha, \beta}$, and $C^{\alpha, \beta}$ in longitudinal geometry for samples with $x=15$ and $3.5 \mathrm{ML}$.

\begin{tabular}{lcccccccc}
\hline \hline & \multicolumn{3}{c}{$x=15 \mathrm{ML}$} & & \multicolumn{3}{c}{$x=3.5 \mathrm{ML}$} \\
\cline { 2 - 5 } \cline { 7 - 8 } & $A^{\alpha, \beta}$ & $B^{\alpha, \beta}$ & $C^{\alpha, \beta}$ & & $A^{\alpha, \beta}$ & $B^{\alpha, \beta}$ & $C^{\alpha, \beta}$ \\
\hline$P_{\text {in }} P_{\text {out }}$ & 170 & 8.3 & 5.0 & & 130 & 4.0 & 11 \\
$S_{\text {in }} P_{\text {out }}$ & 35 & 9.0 & 6.3 & & 46 & 2.7 & 9.0 \\
$P_{\text {in }} S_{\text {out }}$ & 24 & 5.0 & 3.5 & & 33 & 3.6 & 1.7 \\
$S_{\text {in }} S_{\text {out }}$ & 15 & 1.9 & 1.8 & & 12 & 6.9 & 0.95 \\
\hline \hline
\end{tabular}

$$
\begin{gathered}
I_{2 \omega}^{p, p}(\phi, \pm \mathbf{M})=\left|A^{p, p} \pm C^{p, p} \sin 4 \phi\right|^{2}, \\
I_{2 \omega}^{s, p}(\phi, \pm \mathbf{M})=\left|A^{s, p} \pm C^{s, p} \sin 4 \phi\right|^{2}, \\
I_{2 \omega}^{p, s}(\phi, \pm \mathbf{M})=\left| \pm A^{p, s} \pm B^{p, s} \cos 4 \phi\right|^{2}, \\
I_{2 \omega}^{s, s}(\phi, \pm \mathbf{M})=\left| \pm A^{s, s} \pm B^{s, s} \cos 4 \phi\right|^{2},
\end{gathered}
$$

assuming that the effect in the magnetization is weak so that only the zeroth and first order contributions in $\mathbf{M}$ should be accounted for. Here the \pm sign stands in front of the magnetization-induced terms which change sign upon magnetization reversal. The coefficients $A^{i, j}, B^{i, j}, C^{i, j}$ are the combinations of the nonlinear tensor elements as well as Fresnel factors. Note that these patterns do not yield any effect of magnetization reversal for the $S_{\text {in }} S_{\text {out }}$ and $P_{\text {in }} S_{\text {out }}$ MSHG intensity, in contrast to the experimental observation shown in Figs. 3 and 4. They are also unable to properly describe the patterns for the other two polarization combinations [see Fig. 3, dotted lines on the plots for $P_{\text {in }} P_{\text {out }}$ and $S_{\text {in }} P_{\text {out }}$ polarization combinations; also in this case, the Eq. (3) are not able to fit the magnetic contrast because of different symmetry pattern, see below]. Therefore, one has to take into account additional anisotropic contributions to the second-order nonlinear response. In particular, the nonlocal (quadrupole-allowed) contribution from the bulk of a cubic nonmagnetic metals $\left[\mathrm{Cu},{ }^{20} \mathrm{Ag},{ }^{21}\right.$ and $\mathrm{Al}$ (Ref. 22)] and semiconductors [Si (Ref. 23)] has been shown to lead to a fourfold anisotropy of SHG at their (100) surfaces. As shown in Appendix B, accounting for this additional contribution modifies the rotational patterns to

$$
\begin{gathered}
I_{2 \omega}^{p, p}(\phi, \pm \mathbf{M})=\left|A^{p, p}+B^{p, p} \cos 4 \phi \pm C^{p, p} \sin 4 \phi\right|^{2}, \\
I_{2 \omega}^{s, p}(\phi, \pm \mathbf{M})=\left|A^{s, p}+B^{s, p} \cos 4 \phi \pm C^{s, p} \sin 4 \phi\right|^{2}, \\
I_{2 \omega}^{p, s}(\phi, \pm \mathbf{M})=\left| \pm A^{p, s} \pm B^{p, s} \cos 4 \phi+C^{p, s} \sin 4 \phi\right|^{2}, \\
I_{2 \omega}^{s, s}(\phi, \pm \mathbf{M})=\left| \pm A^{s, s} \pm B^{s, s} \cos 4 \phi+C^{s, s} \sin 4 \phi\right|^{2},
\end{gathered}
$$

Since, for our superlattice structures, the normal $z$ direction is not equivalent to the tangential $x, y$ directions, the symmetry of the interior is lower than cubic. Therefore, in the Appendix, the analysis is performed for the $4 / \mathrm{mmm}$ symmetry.

Equation (4) was used for the theoretical fits to the experimental data of Figs. 3 and 4, showing a good agreement between experiment and theory. In Table I, an overview is given of the fitting amplitudes used, indicating the relative

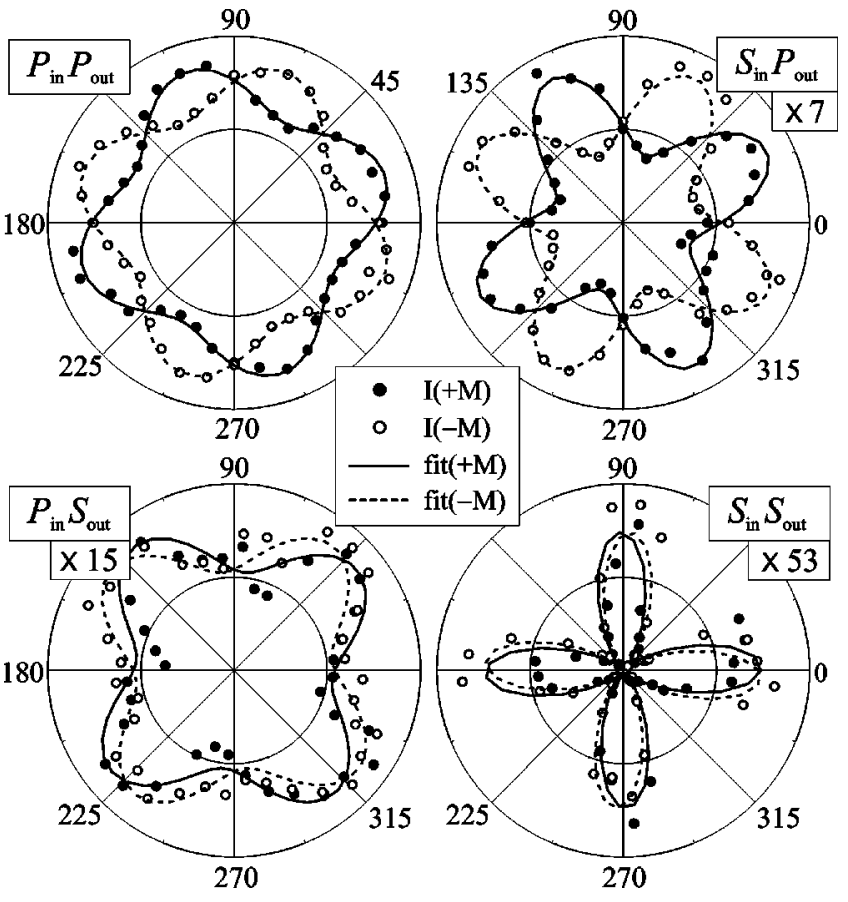

FIG. 5. Rotational anisotropy curves for the sample with single layer thickness of $x=3.5$ monolayers in longitudinal geometry.

strength of the various contributions. In some cases, the quality of the fit was noticeably improved by taking into account the complex character of the azimuthal amplitudes $A^{\alpha, \beta}$, $B^{\alpha, \beta}$, and $C^{\alpha, \beta}$.

For comparison with these results, we have also measured a structure with a small noninteger layer thickness of $x$ $=3.5 \mathrm{ML}$. In that way the ratio between the interface and bulk contribution would be shifted to the former one. In addition, the noninteger thickness might increase the interface roughness thus further increasing the interface MSHG signals. Figure 5 shows the experimental data as well as the fits of the data to Eq. (4) for the sample with $x=3.5 \mathrm{ML}$. Also in this case, the agreement between the theory and experiment is evident. However, no clear influence of the different layer thickness could be seen on the rotational anisotropy curves except that now the relative anisotropic contribution is much stronger for the $S_{\text {in }} S_{\text {out }}$ and much weaker for the $S_{\text {in }} P_{\text {out }}$ polarization combinations.

To summarize, we find that the minimum set of contributions to be taken into account for the data description is (i) the surface/interface (dipole-like) nonmagnetic contribution, (ii) the surface/interface magnetization-induced contribution, and (iii) the nonlocal (quadrupole-allowed) nonmagnetic contribution from the entire region of the sample accessible by light. The quadrupole magnetization-induced contribution, if any, was indistinguishable, by symmetry reasons, from the combination of (ii) and (iii).

\section{ANISOTROPIC NONLINEAR KERR ANGLE}

A slightly different experimental approach to characterize the MSHG response is to measure its polarization dependence with fixed input polarization, as shown in Fig. 6. The 


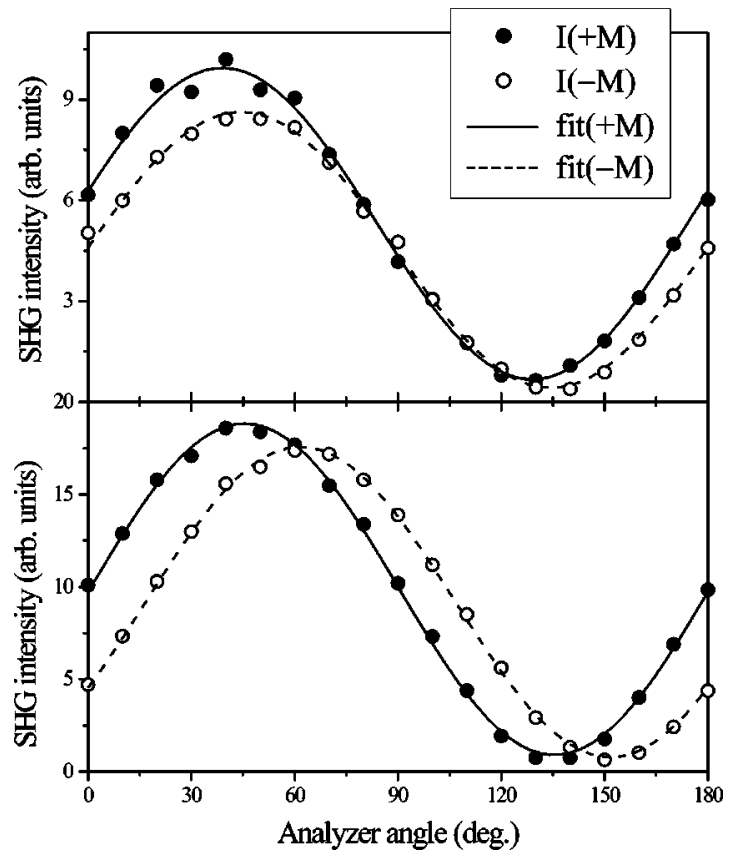

FIG. 6. SHG intensity dependence on the analyzer angle for two different samples: $x=1$ monolayers (top panel) and $x=3.5$ monolayers (bottom panel).

dependence of the detected MSHG intensity on the analyser angle $\psi$ reveals (i) a shift by an angle $\delta \psi$ and (ii) variations of the maxima and minima upon magnetization reversal. Note that the latter is a fingerprint of the MSHG anisotropy since for isotropic surfaces in the longitudinal geometry only the angular shift should be present. ${ }^{24}$ These magnetizationinduced effects can be described as follows. The polarization dependence $I_{2 \omega}^{\alpha, \psi}(\phi, \pm \mathbf{M})$ of the MSHG intensity is given by

$$
I_{2 \omega}^{\alpha, \psi}(\phi)=\left|E_{2 \omega}^{\alpha, p}(\phi, \pm \mathbf{M}) \cos \psi+E_{2 \omega}^{\alpha, s}(\phi, \pm \mathbf{M}) \sin \psi\right|^{2},
$$

where $\alpha$ denotes the incident fundamental polarization ( $s$ or $p)$. The extrema of the $I_{2 \omega}(\psi)$ dependence take place at $\psi$ $=\psi_{ \pm}^{\alpha}$, which obey the condition ${ }^{24}$

$$
\begin{gathered}
\left(\left|E_{2 \omega}^{\alpha, p}(\phi, \pm \mathbf{M})\right|^{2}-\left|E_{2 \omega}^{\alpha, s}(\phi, \pm \mathbf{M})\right|^{2}\right) \tan 2 \psi_{ \pm}^{\alpha} \\
\quad=2 \operatorname{Re}\left[E_{2 \omega}^{\alpha, p}(\phi, \pm \mathbf{M}) E_{2 \omega}^{\alpha, s}(\phi, \pm \mathbf{M})\right] .
\end{gathered}
$$

The relative shift of the extrema upon magnetization reversal is then $\delta \psi^{\alpha}=\psi_{+}^{\alpha}-\psi_{-}^{\alpha}$. In analogy to linear MOKE, we can introduce the nonlinear magneto-optical Kerr angle for anisotropic systems as $\Theta_{\mathrm{K}}^{(2)}=\delta \psi^{\alpha} / 2$. Substituting Eqs. (B9) presented in the Appendix one can clearly see that the Kerr angle depends on the azimuthal angle $\phi$ and also reveals a fourfold pattern. The experimentally derived dependencies of the nonlinear magneto-optical Kerr angle within one quadrant of the whole rotation cycle are shown in Fig. 7. The ellipticity of the MSHG response is determined by the ratio of the maximum and minimum values of the MSHG intensity, which can be obtained by substituting the roots of Eq. (6) into Eq. (5):

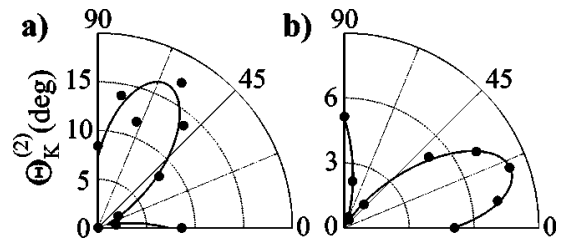

FIG. 7. Nonlinear Kerr angle $\Theta_{K}^{(2)}$ as a function of the sample azimuthal orientation for $S_{\text {in }}$ (a) and $P_{\text {in }}$ (b) input polarizations.

$$
\eta_{\mathrm{K}}^{(2)}=\frac{1}{2}\left[\tan ^{-1}\left[\frac{I_{\mathrm{max}}^{+}}{I_{\min }^{+}}\right]-\tan ^{-1}\left[\frac{I_{\max }^{-}}{I_{\min }^{-}}\right]\right] .
$$

One can also show that the ellipticity is anisotropic with a fourfold symmetry. From our results we find that $\eta_{\mathrm{K}}^{(2)}$ is only about $10 \%$ of the value for $\Theta_{\mathrm{K}}^{(2)}$.

\section{REVEALING SAMPLE SYMMETRY BY ANISOTROPIC MSHG}

Note that the azimuthal rotation patterns (3) and (4) possesses the rotational symmetry

$$
I_{2 \omega}^{\alpha, \beta}\left(\phi, \mathbf{H}_{l}\right)=I_{2 \omega}^{\alpha, \beta}\left(\phi+\frac{\pi}{2}, \mathbf{H}_{l}\right),
$$

and the following mirror symmetry

$$
I_{2 \omega}^{\alpha, \beta}\left(\phi, \mathbf{H}_{l}\right)=I_{2 \omega}^{\alpha, \beta}\left(-\phi,-\mathbf{H}_{l}\right)
$$

where $\alpha, \beta=s, p$ denote fundamental and second harmonic polarizations and $\mathbf{H}_{l}$ is the external longitudinal static magnetic field. The experimental data shown in Figs. 2-5 closely follows the symmetry requirements (8) and (9). The expressions (3) and (4) are derived within certain assumptions about the origin of MSHG. Moreover, we have neglected the linear magneto-optical effects, the magnetic anisotropy, which may lead to a deviation of the direction of the sample magnetization from the direction of the external magnetic field, etc. However, one can formulate more general and model-independent statements about the symmetry of anisotropic MSHG response that do not depend on these factors.

For illustration, we first consider the problem assuming that the fundamental and the harmonic light are plane waves. An $n$-fold symmetric surface rotation of the sample over the angle $2 \pi / n$ leads to an identical crystallographic structure and, independently of the underlying physics of the lightmatter interaction, the results of the MSHG experiment do not change if (i) the sample is also $n$-fold symmetric or the boundary effects can be neglected, and (ii) there is no hysteresis in the magnetic order of the sample upon rotation or reversal of the external magnetic field. Then the field amplitude $E_{2 \omega}^{\alpha, \beta}$ of the plane-wave response obeys

$$
E_{2 \omega}^{\alpha, \beta}(\phi, \mathbf{H})=E_{2 \omega}^{\alpha, \beta}\left(\phi+\frac{2 \pi}{n}, \mathbf{H}\right)
$$


Note that the rotational symmetry (10) holds for any polarization of the fundamental $\alpha$ and MSHG $\beta$ waves, also including any elliptical polarization.

The mirror symmetry requires a more careful analysis. Assume that the plane of incidence of the fundamental light coincides with the $y^{\prime}=0$ plane, that makes angle $\phi$ with respect to the mirror symmetry plane $y=0$ of the sample, as shown in Fig. 1. Now we apply the mirror reflection in the $y=0$ plane to the sample and the fields it is interacting with. For a $P$-polarized fundamental wave the electric field vector possesses nonvanishing $x, y$ and $z$ components, out of which the $y$ component changes sign while the $x$ and $z$ components are unchanged. The $x$ component of the optical magnetic field, as an axial vector, changes sign while the $y$ component is not altered ( $z$ component is zero). One can therefore see that the mirror image of the optical $p$-polarized plane wave is another $p$-polarized plane wave with the same phase and propagating along the positive $x^{\prime \prime}$ direction which makes an angle $-\phi$ relative to the mirror symmetry plane of the sample. Similarly, one can see that the mirror image of the $s$-polarized fundamental wave is another s-polarized plane wave with opposite phase and also propagating along the positive $x^{\prime \prime}$ direction. This phase change of the incident wave is unimportant for second harmonic since the response is quadratic with respect to the fundamental field. The same mirror reflection rules can be found for the outgoing $p$ - and $s$-polarized harmonic fields. The mirror reflection of the external static longitudinal $\mathbf{H}_{l}$ (along $x^{\prime}$ ) and polar $\mathbf{H}_{p}$ (along $z$ ) magnetic fields result in fields along the negative $x^{\prime \prime}$ and $z$ directions, respectively, while the image of the transverse component $\mathbf{H}_{t}$ points along the positive $y^{\prime \prime}$ direction. Thus, within the plane-wave approximation the field of the MSHG response obeys the symmetry

$$
E_{2 \omega}^{\alpha, \beta}\left(\phi, \mathbf{H}_{l}, \mathbf{H}_{p}, \mathbf{H}_{t}\right)=(-1)^{\delta_{\beta, p}} E_{2 \omega}^{\alpha, \beta}\left(-\phi,-\mathbf{H}_{l},-\mathbf{H}_{p}, \mathbf{H}_{t}\right),
$$

where $\delta_{\beta, p}=1$ for $\beta=p$ and 0 for $\beta=s$, if the conditions (i) and (ii) are met and, in addition, (iii) the fundamental and MSHG fields are purely $P$ or $S$ polarized. Note that when the plane of incidence coincides with the mirror symmetry plane $(\phi=0)$ the $s$-polarized component of the MSHG response is purely odd with respect to the applied magnetic field within the plane-wave approximation.

For a real experimental situation the laser spot on the sample has a finite size, often tightly focused. ${ }^{25}$ In this case the electric field is a function of the position on the detector and it is better to consider the total power $W_{2 \omega}^{\alpha, \beta}(\phi, \mathbf{H})$ of the MSHG response, which is the quantity that is measured in a typical MSHG experiment. It is easy to see that the total power obeys the rotational symmetry

$$
W_{2 \omega}^{\alpha, \beta}\left(\phi, \mathbf{H}_{l}\right)=W_{2 \omega}^{\alpha, \beta}\left(\phi+\frac{2 \pi}{n}, \mathbf{H}_{l}\right)
$$

if the conditions (i) and (ii) are met. It also obeys the mirror symmetry

$$
W_{2 \omega}^{\alpha, \beta}\left(\phi, \mathbf{H}_{l}, \mathbf{H}_{p}, \mathbf{H}_{t}\right)=W_{2 \omega}^{\alpha, \beta}\left(-\phi,-\mathbf{H}_{l},-\mathbf{H}_{p}, \mathbf{H}_{t}\right)
$$

if, in addition to requirements (i) and (ii), (iv) the laser spot is either large compared to the wavelength or its shape is symmetric upon mirror reflection in the plane of incidence, and (v) the detector is symmetric relative to the plane of incidence.

Of special interest are the effects of surface/interface roughness and other inhomogeneities in the sample, which have been neglected so far. They lead to light scattering so that the MSHG response is a superposition of many plane waves even if the incident field can be approximated by one plane wave. However, the general symmetry for the rotational pattern of the scattered MSHG intensity still holds if (vi) the number of speckles $N_{\text {speckle }}$ of the scattered field within the detector aperture is large, $N_{\text {speckle }} \gg 1$, and the statistical average of the properties of the inhomogeneities over the irradiated part of the sample obeys the macroscopic sample symmetry.

One can generalize the consideration for other parameters of the MSHG response. For example, one can show that under the requirements discussed above for the rotational symmetry the Kerr angle should obey

$$
\Psi_{\text {Kerr }}^{\alpha}(\phi)=\Psi_{\text {Kerr }}^{\alpha}\left(\phi+\frac{2 \pi}{n}\right) .
$$

The mirror symmetry in longitudinal and polar geometries leads to

$$
\Psi_{\text {Kerr }}^{\alpha}(\phi)=\Psi_{\text {Kerr }}^{\alpha}(-\phi),
$$

so that the extrema in the $\Psi_{\text {Kerr }}^{\alpha}(\phi)$ dependence are reached when the plane of incidence coincides with the mirror symmetry planes of the sample, which is straightforwardly derived from Fig. 7. On the other hand, in the transverse geometry

$$
\Psi_{\text {Kerr }}^{\alpha}(\phi)=-\Psi_{\text {Kerr }}^{\alpha}(-\phi),
$$

so that the transverse Kerr angle vanishes when the plane of incidence coincides with the mirror symmetry plane for exact $p$ - and $s$-polarized fundamental waves. There is no effect of the mirror symmetry on the $\Psi_{\text {Kerr }}^{\alpha}(\phi)$ dependence if the external magnetic field possesses nonvanishing projections on both the plane of incidence (polar and/or longitudinal components) and its normal (transverse component).

Therefore, we have formulated the conditions which are needed to observe certain rotational and mirror symmetries of the anisotropic MSHG response (its azimuthal intensity patterns, magneto-optical Kerr angles, etc.). These symmetries reveal the symmetry of the sample. We have found that additional requirements on the symmetry of the experimental setup should be fulfilled to observe the mirror symmetry. Although the consideration is performed mostly for linear $s$ and $p$ polarizations, it can be straightforwardly generalized to include other polarizations. For example, for circular light polarization the mirror symmetry must be supplemented by a reversal of the light helicity. In a more general case of an elliptical polarization, the tilt of the ellipse axis must also be reversed. 


\section{CONCLUSION}

In conclusion, we have shown that the MSHG response of $\mathrm{Fe} / \mathrm{Au}(001)$ superlattices shows a strong azimuthal anisotropy on both the MSHG intensity as well as in the nonlinear magneto-optical Kerr rotation. These observations can fully be described by taking into account not only the interfaceallowed dipole contributions but in addition the higher order (bulk-like) quadrupole contributions. This result is fully consistent with observations from other nonmagnetic (001) surfaces, but was not a priori evident, as in principle dipoleallowed magnetic contribution alone could provide the necessary anisotropy. In addition, we have shown that, independent of the details of the nonlinear optical response, very general and powerful statements can be made that relate the observed MSHG response with the magnetic and crystallographic symmetries. This is in line with similar observations that were recently made by Fiebig et al. using MSHG to solve the symmetry of the spin ordering in several antiferromagnetically ordered crystals ${ }^{26}$ that could not be solved by neutron scattering. This shows once more that MSHG is indeed a powerful tool to reveal the crystallographic and magnetic symmetry of spin ordered systems. A direct extension of this work would be to look at the symmetry of the MSHG fields instead of the intensities, which can be done by measuring the phase of the optical signals as well. ${ }^{27,28}$

\section{ACKNOWLEDGMENTS}

This work was partly supported by the TMR network NOMOKE.

\section{APPENDIX A: SURFACE AND INTERFACE CONTRIBUTIONS}

First we focus our attention on the contributions to the MSHG response from the top surface and the internal interfaces in the $\mathrm{Fe} / \mathrm{Au}$ superlattice. At surfaces and interfaces, the properties of a solid change abruptly, leading to a strong contribution to the second-order nonlinear optical response due to asymmetry of electronic wave functions and fast variations of the optical fields on a very short spatial scale of the order of the interatomic distance. A microscopic approach to solve the nonlinear interaction of light with the medium at surfaces and interfaces is therefore a complex problem which intrinsically involves the surface nonlocal screening. ${ }^{29}$ On the other hand, since the wavelength of light is large on the microscopic scale, the result can be expressed in terms of a surface (interface) dipole-like nonlinear susceptibility $\chi^{(2), S}$, which is a third-rank tensor. ${ }^{29-31}$

In a superlattice, one has the top surface and many interfaces which can contribute to the second-order response. Note, however, that there is a strong cancellation expected between neighboring interfaces $I_{1}$ and $I_{2}$ due to their opposite orientation, leading to

$$
\chi^{(2), I_{1}} \approx-\chi^{(2), I_{2}}
$$

where $\chi^{(2), I_{1}}$ and $\chi^{(2), I_{2}}$ are the nonlinear susceptibilities of the two interfaces. A nonvanishing contribution of the interfaces to the MSHG response can then arise due to two fac- tors. (i) The cancellation may be incomplete: $\chi^{(2), I_{1}}$ $+\chi^{(2), I_{2}} \neq 0$ because of a slight difference between the "upward" and "downward" interfaces due to, e.g., growthinduced variations of the crystallographic structure of the interfaces of the two types. Also, due to the extended character of the electron wave functions, the top surface may induce effects on the electronic structure of buried interfaces, which can be different for $I_{1}$ and $I_{2}$. (ii) The fully antisymmetric part of the interface susceptibilities, $\left(\chi^{(2), I_{1}}\right.$ $\left.-\chi^{(2), I_{2}}\right) / 2$, can also contribute to the total response due to a small difference in the local optical fields, which are retarded and attenuated at the lower interface.

Since the thickness of the layers in the superlattice is very small relative to the optical wavelength, one can introduce macroscopically averaged fields and nonlinear polarizations and replace the interior of the sample by a uniform medium with effective parameters. Within this effective-medium approach the contributions to the MSHG response can then be described in terms of (i) dipole-like susceptibility $\chi^{S}$ of the top surface, (ii) dipole-like susceptibility $\chi^{I, d}$ due to incomplete cancellation of the interface susceptibilities and (iii) a nonlocal (quadrupole-like) contribution which arises from the fully antisymmetric part of the interface susceptibilities due to spatial variation of the macroscopic effective field $\mathbf{E}^{\mathrm{eff}}(\omega)$ along $z$

$$
P_{i}^{I, Q}(2 \omega)=\chi_{i j z l}^{I, Q} E_{j}^{\mathrm{eff}}(\omega) \nabla_{z} E_{l}^{\mathrm{eff}}(\omega)
$$

Note that the derivatives of $\mathbf{E}^{\mathrm{eff}}(\omega)$ along the layers do not enter Eq. (A2) since within the plane-wave approximation the spatial variation of $\mathbf{E}^{\mathrm{eff}}(\omega)$ in the tangential direction is fully determined by the tangential projection of the wave vector of the incident wave through the whole sample. In this section we focus on the dipole-like contributions of the top surface and interfaces while the contribution due to Eq. (A2) is discussed in Appendix B.

Within the plane-wave approximation the amplitude of the $\alpha$-polarized MSHG response induced by $\beta$-polarized fundamental light $(\alpha, \beta=s, p)$ arising via the dipole-like nonlinear susceptibilities can be written as

$$
E_{2 \omega}^{\alpha, \beta}=\sum_{i^{\prime}, j^{\prime}, k^{\prime}} \chi_{i^{\prime} j^{\prime} k^{\prime}} \widetilde{F}_{i^{\prime}}^{\alpha}(2 \omega) F_{j^{\prime}}^{\beta}(\omega) F_{k^{\prime}}^{\beta}(\omega) E_{0}^{\beta}(\omega)^{2},
$$

where $\widetilde{F}_{i^{\prime}}^{\alpha}(2 \omega)$ and $F_{j^{\prime}}^{\beta}(\omega)$ are the Fresnel factors, $E_{0}(\omega)$ is the amplitude of the incident wave and $\chi_{i^{\prime} j^{\prime} k^{\prime}}$ denotes elements of the total effective dipole susceptibility of the top surface and the interfaces in the laboratory frame.

The linear magneto-optical effects are usually weak compared to those in the nonlinear optical response so that the effect of the magnetization on the Fresnel factors can be neglected. The Fresnel factors can also be assumed isotropic (independent of $\phi$ ) so that $F_{y^{\prime}}^{p}=F_{x^{\prime}}^{s}=F_{z}^{s}=0$ and the dependencies on the azimuthal angle $\phi$ and the direction of the magnetization $\mathbf{M}$ solely arise from the nonlinear susceptibility $\chi_{i^{\prime} j^{\prime} k^{\prime}}(\phi, \mathbf{M})$. Since the effect of the magnetic order on 
the electronic properties is weak the magnetizationdependence of the dipole susceptibility can be expanded in powers of $\mathbf{M}$ as

$$
\chi_{i j k}(\mathbf{M})=\chi_{i j k}(0)+X_{i j k l} M_{l}+\cdots,
$$

where $\mathbf{X}$ is an axial fourth-rank tensor. Below we use the linear-in-M approximation and neglect the higher-order terms.

For a fourfold symmetric sample the nonmagnetic $\chi(0)$ part of the susceptibility possesses three independent elements with $z z z, z\|\|$, and \|\|$z$ indices, where $\|$ stand for the in-plane $x$ or $y$ coordinates. This tensor is purely isotropic and does not lead to rotational anisotropy. Its contribution to the $s$-polarized response vanishes for both $p$ - and $s$-polarized fundamental waves ( $p, s$ and $s, s$ response, respectively). All three components of the $\chi(0)$ tensor contribute to the response for the $p, p$ polarization combination while only the $z\|\|$ element contributes to the $s, p$ response.

In the longitudinal geometry the magnetization vector is within the surface plane. Thus, in the crystallographic frame we need the components of the tensor $X_{i j k l}$ with $l=x$ or $y$. For a fourfold symmetric sample they are

$$
\begin{gathered}
X_{x x y x}=-X_{y y x y}, \quad X_{y x x x}=-X_{x y y y}, \\
X_{y y y x}=-X_{x x x y}, \quad X_{y z z x}=-X_{x z z y}, \\
X_{z z y x}=-X_{z z x y} .
\end{gathered}
$$

The elements connected to the first and the last elements in Eq. (A5) by the simple permutation symmetry $X_{i j k l} \equiv X_{i k j l}$ are omitted.

For $s, s$ MSHG the fundamental field and the nonlinear response are along $y^{\prime}$ while the longitudinal magnetization is along $x^{\prime}$. Thus the magnetization-induced response arises via

$$
\chi_{y^{\prime} y^{\prime} y^{\prime}}^{m}=X_{y^{\prime} y^{\prime} y^{\prime} x^{\prime}} M_{x^{\prime}}=\left[X_{y y y x}+\frac{\cos 4 \phi-1}{4} \zeta_{m}\right] M_{x^{\prime}},
$$

where $\chi_{i^{\prime} j^{\prime} k^{\prime}}^{m}$ denotes the laboratory frame element of the nonlinear susceptibility arising due to the second term on the right-hand side of Eq. (A4), and

$$
\zeta_{m}=X_{y y y x}-X_{y x x x}-2 X_{x x y x} .
$$

For $s, p$-MSHG the relevant magnetization-induced component is

$$
\chi_{x^{\prime} y^{\prime} y^{\prime}}^{m}=X_{x^{\prime} y^{\prime} y^{\prime} x^{\prime}} M_{x^{\prime}}=\frac{1}{4} \zeta_{m} \sin 4 \phi M_{x^{\prime}},
$$

while $\chi_{z^{\prime} y^{\prime} y^{\prime}}^{m}=0$.

Analogously, for $p, s$-MSHG we find

$$
\begin{gathered}
\chi_{y^{\prime} x^{\prime} x^{\prime}}^{m}=X_{y^{\prime} x^{\prime} x^{\prime} x^{\prime}} M_{x^{\prime}}=\left[X_{y x x x}+\frac{1-\cos 4 \phi}{4} \zeta_{m}\right] M_{x^{\prime}}, \\
\chi_{y^{\prime} z z}^{m}=X_{y^{\prime} z z x^{\prime}} M_{x^{\prime}}=X_{y z z x} M_{x^{\prime}},
\end{gathered}
$$

while $\chi_{y^{\prime} x^{\prime} z}^{m}=0$.

Finally, for $p, p$-MSHG we have

$$
\chi_{x^{\prime} x^{\prime} x^{\prime}}^{m}=X_{x^{\prime} x^{\prime} x^{\prime} x^{\prime}} M_{x^{\prime}}=-\frac{1}{4} \zeta_{m} \sin 4 \phi M_{x^{\prime}},
$$

with $\chi_{x^{\prime} x^{\prime} z}^{m}=\chi_{z x^{\prime} x^{\prime}}^{m}=\chi_{x^{\prime} z z}^{m}=\chi_{z z x^{\prime}}^{m}=\chi_{z z z}^{m}=0$.

By substituting Eqs. (A6)-(A11) into Eq. (A3) and adding the isotropic contribution of the first term on the righthand side of Eq. (A4) one can see that the azimuthal dependence of the response has the form

$$
\begin{gathered}
E_{2 \omega}^{p, p}\left(\phi, \pm \mathbf{M}_{l}\right)=A^{p, p} \pm C^{p, p} \sin 4 \phi, \\
E_{2 \omega}^{s, p}\left(\phi, \pm \mathbf{M}_{l}\right)=A^{s, p} \pm C^{s, p} \sin 4 \phi, \\
E_{2 \omega}^{p, s}\left(\phi, \pm \mathbf{M}_{l}\right)= \pm A^{p, s} \pm B^{p, s} \cos 4 \phi, \\
E_{2 \omega}^{s, s}\left(\phi, \pm \mathbf{M}_{l}\right)= \pm A^{s, s} \pm B^{s, s} \cos 4 \phi,
\end{gathered}
$$

where the \pm sign indicates those terms which change their sign upon magnetization reversal, $A, B$ and $C$ are independent of $\phi$ and the direction of the longitudinal magnetization $\mathbf{M}_{l}$. Since the light intensity is related to the field via $I_{2 \omega}^{\alpha, \beta}$ $=c /(2 \pi)\left|E_{2 \omega}^{\alpha, \beta}\right|^{2}$, we arrive to Eq. (3), where the $A, B$ and $C$ amplitudes are redefined to include the $\sqrt{c /(2 \pi)}$ prefactor.

Note that the anisotropic amplitudes arise via a single combination (A7) of the elements of the $\mathbf{X}$ tensor so that their relative size is purely determined by the Fresnel factors. For highly refractive media, $|\epsilon(\omega)| \gg 1$ and $|\epsilon(2 \omega)| \gg 1$ and relatively small angles of incidence, the Fresnel factors $F_{x^{\prime}}^{p}$ and $F_{y}^{s}$, for tangential fields are close to each other so that the following approximate relation

$$
\left|B^{s, s}\right| \approx\left|B^{p, s}\right| \approx\left|C^{s, p}\right| \approx\left|C^{p, p}\right|
$$

is expected.

\section{APPENDIX B: NONLOCAL CONTRIBUTIONS}

Here we consider the nonlocal contribution to the secondorder response within the effective medium approximation. Although both $\mathrm{Au}$ and bcc-Fe are cubic crystals, in the superlattice the wave functions have lower $4 / \mathrm{mmm}$ symmetry since the normal $z$ direction is not anymore equivalent to the in-plane $x$ and $y$ directions. To the first order in nonlocality (quadrupole) the effective nonlinear polarization is proportional to the gradient of the fundamental field

$$
P_{i}(2 \omega)=\chi_{i j k l}^{Q}(\mathbf{M}) E_{j}(\omega) \nabla_{k} E_{l}(\omega) .
$$

This nonlinear source includes the nonlocal contributions from the interior of the layers and the fully asymmetric part of the response of the interfaces (A2) as discussed in the previous section. Similar to Eq. (A4), we expand the $\chi_{i j k l}^{Q}(\mathbf{M})$ as

$$
\chi_{i j k l}^{Q}(\mathbf{M})=\chi_{i j k l}^{Q}(0)+X_{i j k l m}^{Q} M_{m}+\cdots .
$$

Below we take only the first (nonmagnetic) term of the expansion as it provides enough freedom to fully describe the 
experimental data. Thus, in this section we completely neglect the effect of the magnetic order on the nonlocal contribution and omit " $(0)$ " for notation simplicity.

For the $4 / \mathrm{mmm}$ symmetry class the independent elements of the $\chi^{Q}$ tensor are

$$
\begin{gathered}
\chi_{x x y y}^{Q}=\chi_{y y x x}^{Q} \\
\chi_{x y x y}^{Q}=\chi_{y x y x}^{Q}, \\
\chi_{x x x x}^{Q}=\chi_{y y y y}^{Q}, \\
\chi_{x x z z}^{Q}=\chi_{y y z z}^{Q} \\
\chi_{z z x x}^{Q}=\chi_{z z y y}^{Q} \\
\chi_{x z x z}^{Q}=\chi_{y z y z}^{Q}, \\
\chi_{z x z x}^{Q}=\chi_{z y z y}^{Q}, \\
\chi_{z z z z}^{Q} .
\end{gathered}
$$

Note that the fourfold anisotropy may arise only from the first three elements with purely tangential components and we focus our attention on them. The other components do not change upon azimuthal rotation and contribute only to the $A^{s, p}$ and $A^{p, p}$ amplitudes.

For $s, s-$ SHG the anisotropic contribution arises via the $y^{\prime} y^{\prime} x^{\prime} y^{\prime}$ element of the $\chi^{Q}$ tensor which is given in the laboratory frame by

$$
\chi_{y^{\prime} y^{\prime} x^{\prime} y^{\prime}}^{Q}=\frac{1}{4} \zeta \sin 4 \phi
$$

where

$$
\zeta=\chi_{x x x x}^{Q}-\chi_{x y x y}^{Q}-2 \chi_{x x y y}^{Q}
$$

The anisotropic part of $s, p-\mathrm{SHG}$ arises via

$$
\chi_{x^{\prime} y^{\prime} x^{\prime} y^{\prime}}^{Q}=\chi_{x y x y}^{Q}+\frac{1-\cos 4 \phi}{4} \zeta .
$$

For $p, s-\mathrm{SHG}$ one finds

$$
\chi_{y^{\prime} x^{\prime} x^{\prime} x^{\prime}}^{Q}=-\frac{1}{4} \zeta \sin 4 \phi
$$

Finally, for $p, p-\mathrm{SHG}$ we have

$$
\chi_{x^{\prime} x^{\prime} x^{\prime} x^{\prime}}^{Q}=\chi_{x x x x}^{Q}+\frac{\cos 4 \phi-1}{4} \zeta .
$$

Adding the anisotropic contributions due to Eqs. (B4)(B8) and the isotropic components of the nonlocal response, one can see that the azimuthal dependence of the response field amplitude now reads as

$$
\begin{gathered}
E_{2 \omega}^{p, p}\left(\phi, \pm \mathbf{M}_{l}\right)=A^{p, p}+B^{p, p} \cos 4 \phi \pm C^{p, p} \sin 4 \phi, \\
E_{2 \omega}^{s, p}\left(\phi, \pm \mathbf{M}_{l}\right)=A^{s, p}+B^{s, p} \cos 4 \phi \pm C^{s, p} \sin 4 \phi, \\
E_{2 \omega}^{p, s}\left(\phi, \pm \mathbf{M}_{l}\right)= \pm A^{p, s} \pm B^{p, s} \cos 4 \phi+C^{p, s} \sin 4 \phi, \\
E_{2 \omega}^{s, s}\left(\phi, \pm \mathbf{M}_{l}\right)= \pm A^{s, s} \pm B^{s, s} \cos 4 \phi+C^{s, s} \sin 4 \phi,
\end{gathered}
$$

where the new nonmagnetic anisotropic amplitudes originate from a single combination (B5) of the elements of the $\chi^{Q}$ tensor and for a highly refractive medium and not too large angle of incidence

$$
\left|C^{s, s}\right| \approx\left|C^{p, s}\right| \approx\left|B^{s, p}\right| \approx\left|B^{p, p}\right|
$$

is expected.
*On leave from the Institute of Crystallography, 117234 Moscow, Russia.

†Corresponding author. Email address: kirilyuk@sci.kun.nl

${ }^{1}$ R.-P. Pan, H. D. Wei, and Y. R. Shen, Phys. Rev. B 39, 1229 (1989).

${ }^{2}$ J. Reif, J. C. Zink, C. M. Schneider, and J. Kirschner, Phys. Rev. Lett. 67, 2878 (1991).

${ }^{3}$ Nonlinear Optics in Metals, edited by K. H. Bennemann (Clarendon Press, Oxford, 1998).

${ }^{4}$ M. Fiebig, D. Fröhlich, B. B. Krichevtsov, and R. V. Pisarev, Phys. Rev. Lett. 73, 2127 (1994).

${ }^{5}$ M. Fiebig, D. Fröhlich, K. Kohn, St. Leute, Th. Lottermoser, V. V. Pavlov, and R. V. Pisarev, Phys. Rev. Lett. 84, 5620 (2000).

${ }^{6}$ H. A. Wierenga, W. de Jong, M. W. J. Prins, Th. Rasing, R. Vollmer, A. Kirilyuk, H. Schwabe, and J. Kirschner, Phys. Rev. Lett. 74, 1462 (1995).

${ }^{7}$ M. Straub, R. Vollmer, and J. Kirschner, Phys. Rev. Lett. 77, 743 (1996).

${ }^{8}$ B. Koopmans, M. Groot Koerkamp, Th. Rasing, and H. van den Berg, Phys. Rev. Lett. 74, 3692 (1995).

${ }^{9}$ T. M. Crawford, C. T. Rogers, T. J. Silva, and Y. K. Kim, Appl. Phys. Lett. 68, 1573 (1996).
${ }^{10}$ A. Kirilyuk, Th. Rasing, R. Mégy, and P. Beauvillain, Phys. Rev. Lett. 77, 4608 (1996).

${ }^{11}$ V.V. Pavlov, R.V. Pisarev, A. Kirilyuk, and Th. Rasing, Phys. Rev. Lett. 78, 2004 (1997).

${ }^{12}$ A.V. Petukhov, I.L. Lyubchanskii, and Th. Rasing, Phys. Rev. B 56, 2680 (1997).

${ }^{13}$ K. Takanashi, S. Mitani, M. Sato, H. Fujimori, H. Nakajima, and A. Osawa, Appl. Phys. Lett. 67, 1016 (1995).

${ }^{14}$ S. Mitani, K. Takanashi, H. Nakajima, K. Sato, R. Schreiber, P. Grunberg, and H. Fujimori, J. Magn. Magn. Mater. 156, 7 (1996).

${ }^{15}$ K. Sato, J. Abe, H. Ikekame, K. Takanashi, S. Mitani, and H. Fujimori, J. Magn. Soc. Jpn. 20, (Suppl. S1), 35 (1996).

${ }^{16}$ K. Sato, E. Takeda, M. Akita, M. Yamaguchi, K. Takanashi, S. Mitani, H. Fujimori, and Y. Suzuki, J. Appl. Phys. 86, 4985 (1999).

${ }^{17}$ A. Kirilyuk, V. V. Pavlov, R. V. Pisarev, and Th. Rasing, Phys. Rev. B 61, R3796 (2000).

${ }^{18}$ H. Nakazawa, S. Mitani, K. Takanashi, H. Nakajima, A. Osawa, and H. Fujimori, J. Magn. Soc. Jpn. 20, 353 (1996) (in Japanese). 
${ }^{19}$ W. Hübner and K. H. Bennemann, Phys. Rev. B 40, 5973 (1989).

${ }^{20}$ R. Vollmer, M. Straub, and J. Kirschner, Surf. Sci. 352, 684 (1996).

${ }^{21}$ D. A. Koos, V. L. Shanon, and G. L. Richmond, Phys. Rev. B 47, 4730 (1993).

${ }^{22}$ K. Pedersen and O. Keller, J. Opt. Soc. Am. B 6, 2412 (1989).

${ }^{23}$ H. W. K. Tom, T. F. Heinz, and Y. R. Shen, Phys. Rev. Lett. 51, 1983 (1983).

${ }^{24}$ Th. Rasing, M. Groot Koerkamp, B. Koopmans, and H. van den Berg, J. Appl. Phys. 79, 6181 (1996).

${ }^{25}$ Note that for spot sizes comparable to the wavelength, fundamental light intensity variations on the sides may lead to additional gradients of the optical field and, thus, additional contributions to the response: O. A. Aktsipetrov (private communication).

${ }^{26}$ M. Fiebig, D. Fröhlich, K. Kohn, St. Leute, Th. Lottermoser, V. V. Pavlov, and R. V. Pisarev, Phys. Rev. Lett. 84, 5620 (2000).

${ }^{27}$ R. Stolle, K. J. Veenstra, F. Manders, Th. Rasing, H. A. M. van den Berg, and N. Persat, Phys. Rev. B 55, R4925 (1997).

${ }^{28}$ K. J. Veenstra, A. V. Petukhov, A. P. de Boer, and Th. Rasing, Phys. Rev. B 58, R16020 (1998).

${ }^{29}$ A. Liebsch, Electronic Excitations at Metal Surfaces (Plenum, New York, 1997).

${ }^{30}$ J. E. Sipe, D. J. Moss, and H. M. van Driel, Phys. Rev. B 35, 1129 (1987).

${ }^{31}$ A. V. Petukhov, V. L. Brudny, W. L. Mochán, J. A. Maytorena, B.S. Mendoza, and Th. Rasing, Phys. Rev. Lett. 81, 566 (1998). 